\title{
Rate of Convergence of the Sine Imprecise Functions
}

\author{
Kangujam Priyokumar Singh \\ Department of Mathematical Sciences, Bodoland University, Kokrajhar BTAD, Assam, India \\ E-mail: pk_mathematics@yahoo.co.in \\ *Sahalad Borgoyary \\ Department of Basic Sciences (Mathematics), Central Institute of Technology Kokrajhar BTAD, Assam, India \\ (Corresponding author) E-mail: s.borgoyary@cit.ac.in/borgsaha@gmail.com
}

\begin{abstract}
We convert polynomial function of degree $\mathrm{n}^{\text {th }}$ into imprecise form to obtain an important point called conversion point. For some particular region, we collect the finite number of data points to obtain the most economical function called imprecise function. Conversion point of the functions is shown with the help of MUPAD graph. Further we study the area of the imprecise function occurred by the multiplication of sine function to know how much variation of the imprecise functions are obtained for the respective intervals. For different imprecise polynomial we study level of the rate of convergence.
\end{abstract}

Index Terms - Rate of Convergence, Imprec ise Function, Conversion Point, Imprecise Number, Diversion Point, Imprecise Polynomials.

\section{INTRODUCTION}

Imprecise number is an extension definition of fuzzy number. The term was first used in the article theory of Believes and Realities [6]. Collection of all these numbers is called imprecise set. This set has character same as to classical set. Impreciseness of the objects are always formed an imprecise number in a certain interval. Where the impreciseness is the membership function obtained by an object in the extension definition of fuzzy set. If we take the union of infinite intervals then it will create a function called imprecise function. Thus study of the behavior of this type of function is very important. It will help us to solve many difficult problems. For example behavior of a loudness of sound produces from a device is non-imprecise function form. So, it can be converted into audible form by the help of multiplication of sine function. This level of the function is generally known as normal imprecise function.

Our problems are not always expressible into general function form. In most of the time it is obtained only into the imprecise form. As for example receivers are getting response through the frequency of the wave produces from a main places called server. This frequency has character like imprecise function. When it is completely imprecise form, then it will be functioned, otherwise will not be run properly. This phenomenon is known as controllable and non-controllable. When it is uncontrollable form, we will minimize the force produce from the main server so that it can be transformed into controllable form. This experiment is doing available in the field of science and technology. The value of the level for which a function is either controllable or not is called rate of convergence. Thus the rate of convergence is also play an important in the field of application. If we can identify this value, then what amount of force or energy is required for a particular problem can be easily identified. This value is definable by the help of the imprecise function. So any polynomial is converted into imprecise function so that we can control it according to our needs.

Imprecise function which is obtained by the multiplication of some other functions is called multiplication factor. Thus any continuous function is multiplied by sine or cosine function to obtain imprecise polynomials. For a certain region we will collect finite number of data collection of points. And with the help of these points we will identify most valuable function or the controllable function called imprecise function for these points.

Area bounded by the imprecise function help us to know how the variation of the effect of impreciseness of an object is occurred for the different intervals. For this reason we define formula of the area of an imprecise number in the general summation form. Thus the area formulae of the different angles of sine functions are also play an important role in this article.

The remaining sections of the article are divided into the following ways:

Section II is contain about the preliminary definition of imprecise function, section III is contain definition of new function called imprecise function, Section IV is contain conversion point obtain by the help of mu ltip lication of the sine and cosine function, Section V is contain rate of convergence of different imprecise function so that we can study variation of the effect of imprecise for the respective intervals. Finally section VI is the conclusion and the summary of this article. 


\section{PRELIMINARIES}

Before starting this article it is necessary to recall the definition of imprecise number, partial presence, membership value etc. over the real line which are discussed in the article [9],[10],[12] [13], [15] and [16].

\section{A. Imprecise Number:}

Imprecise number is a closed interval $\mathrm{N}=[\alpha, \beta, \gamma]$ which is divided into closed sub-intervals with the partial presence of element $\beta$ in both the intervals.

\section{B. Partial presence:}

Partial presence of an element in an imprecise real number $[\alpha, \beta, \gamma]$ is described by the present level indicator function $p(x)$ which is counted from the reference function $r(x)$ such that present level indicator for any $\mathrm{x}, \alpha \leq x \leq \gamma$, is $(p(x)-r(x))$, where $0 \leq$ $r(x) \leq p(x) \leq 1$

\section{Membership value:}

If an imprecise number $N=[\alpha, \beta, \gamma]$ is associated with a presence level indicator function $\mu_{N}(x)$ such that,

$$
\mu_{N}(x)=\left\{\begin{array}{rc}
\mu_{1}(x), & \text { when } \alpha \leq x \leq \beta \\
\mu_{2}(x), & \text { when } \beta \leq x \leq \gamma \\
0, & \text { otherwise }
\end{array}\right.
$$

With a constant reference function 0 in the entire real line. Where $\mu_{1}(x)$ is continuous and non-decreasing in the interval $[\alpha, \beta]$, and $\mu_{2}(x)$ is a continuous and nonincreasing in the interval $[\beta, \gamma]$ with

$$
\mu_{1}(\alpha)=\mu_{2}(\gamma)=0
$$

then $\left(\mu_{1}(\beta)-\mu_{2}(\beta)\right)$ is called membership value of the indicator function $\mu_{N}(x)$

\section{Normal Imprecise Number:}

A normal imp recise number $N=[\alpha, \beta, \gamma]$ is associated with a presence level indicator function $\mu_{N}(x)$ such that,

$$
\mu_{N}(x)=\left\{\begin{array}{cc}
\mu_{1}(x), & \text { when } \alpha \leq x \leq \beta \\
\mu_{2}(x), & \text { when } \beta \leq x \leq \gamma \\
0, & \text { otherwise }
\end{array}\right.
$$

With a constant reference function 0 in the entire real line. Where $\mu_{1}(x)$ is continuous and non-decreasing in the interval $[\alpha, \beta]$ and $\mu_{2}(x)$ is a continuous and nonincreasing in the interval $[\beta, \gamma]$ with

$$
\begin{aligned}
& \mu_{1}(\alpha)=\mu_{2}(\gamma)=0 \\
& \mu_{1}(\beta)=\mu_{2}(\beta)=1
\end{aligned}
$$

Here, the imprecise number is characterized by $\left\{x, \mu_{N}(x), 0: x \in R\right\}, \mathrm{R}$ being the real line.

For any real line, $0 \leq \mu_{1}(x) \leq \mu_{2}(x) \leq 1$ normal and subnormal imprecise number will be characterized in common, $\left\{x, \mu_{1}(x), \mu_{2}(x): x \in R\right\}$, where $\mu_{1}(x)$ is called membership function measured from the reference function $\mu_{2}(x)$ and $\left(\mu_{1}(x)-\mu_{2}(x)\right)$ is called the membership value of the indicator function.

Here, the number is normal imprecise number when me mbership value of indicator function $\mu_{N}(x)$ is equal to 1 otherwise subnormal if not equal to 1 . Moreover if the membership value of $\mu_{N}(x)$ is equal to 1 then the set is universal set and the null or empty set if the value of $\mu_{N}(x)$ is equal to 0 .

\section{IMPRECISE FUNCTIONS}

Imprecise number is an interval definable number where the interval is an area bounded by some section of a function formed by the impreciseness of objects. So, the function which contains such type of imprecise intervals within in it is known imprecise function. At present this phenomenon is occurring available in the field of science and technology. For example possible of impreciseness of electromagnetic wave frequency in between the server and the receiver is an imprecise function. Where, the finite numbers of imprecise numbers are occurred in between the locations. So, the bigger function to connect these two locations is an imprecise function. From this point of view it can be introduced some conditions for the imprecise functions as follows:

(i) Function must be continuous in some region

(ii) Function should be oscillation in nature

(iii) Function must have finite number of maximum and minimum values within the interval.

(iv) Function should be semi-periodic/periodic in nature.

Generally, formation of effect of the impreciseness in the form of sine and cosine functions for a certain region is interval definable number. Thus, effect of any function which is defined for any intervals can be converted into imprecise function with the help of multiplication factors of sine and cosine functions.

\section{A. Conversion point-}

It is a point from where non-imprecise function starts to convert into imprecise function. If we can identify this point, then we will get easily their applications. As for example loudness of sound causes brain and heart diseases because of non-imprecise function of character produces from a sound device. So, it may be possible to control by converting them into human ear audible level by the help of volu me regulatory. Further in present most of the times ultraviolet ray reaches in the ground is nonimprecise function form due to lake of the friction of Ozone layer caused by the environmental pollutions. This phenomenon is creating problems in the life of living being causing skin disease etc. Thus if we can invent a new device having Ozone layer character, then it will save us from these type of problems. This may be possible due to the multiplication factor which can convert any function into imprecise function form. 
Normally these phenomena are already applied in many of the experiment like volume regulator or the regulators used in the different fields for their practical purposes.

\section{CONVERSION OF POLYNOMIALS INTO IMPRECISE FUNCTIONS}

There are many polynomials, which are raised to infinite direction without coming back to the ground level. So it is called uncontrolled function. But this type of function can also be transformed into oscillation form to meet the ground level repeatedly from a certain point. This point is known as conversion point. This phenomenon is occurred by the multiplication of a function which can convert into imprecise function form. This multipliable function is called multiplication factors.

Thus the imprecise function obtained by the multiplication of sine function is known as sine imprec ise function and the imprecise function obtained by the multiplication of cosine is called cosine imprecise function.

For this purpose, let us consider

$$
\begin{gathered}
p_{1}\left(x_{1}, y_{1}\right), p_{2}\left(x_{2}, y_{2}\right), p_{3}\left(x_{3}, y_{3}\right) \ldots \ldots \ldots \ldots \ldots . . \\
\ldots \ldots \ldots \ldots \ldots p_{n}\left(x_{n+1}, y_{n+1}\right)
\end{gathered}
$$

be the $n^{\text {th }}$ collection of points, which are above and below the given polynomial of degree $n$,

$$
y=c_{0}+c_{1} x+c_{2} x^{2}+c_{3} x^{3} \ldots \ldots .+c_{n} x^{n}
$$

To convert it into imprecise form, let us multiply this polynomial by a sine function.

Thus,

$$
\begin{gathered}
y=\left(c_{0}+c_{1} x+c_{2} x^{2}+c_{3} x^{3}+\right. \\
\left.\ldots \ldots \ldots . . .+c_{n} x^{n}\right) \sin (l x) ; l \in Z
\end{gathered}
$$

will be a sine imprecise polynomial of degree $n$.

To obtain standard imprecise polynomial averagely passing closed to these points, we follow the rules of matrix multiplication as follows:

As, the point $p_{1}, p_{2}, \ldots \ldots \ldots \ldots \ldots p_{n}$ are satisfied the given polynomial. By the law of roots, we will get $n^{\text {th }}$ set of simultaneous linear equations having arbitrary constants $c_{0}, c_{1}, c_{2}, \ldots \ldots \ldots \ldots \ldots$.

$$
\begin{gathered}
\ldots \ldots \ldots, c_{n} \text { as follows: } \\
y_{1}=\left(c_{0}+c_{1} x_{1}+c_{2} x_{1}{ }^{2}+c_{3} x_{1}{ }^{3}\right. \\
\left.\ldots \ldots \ldots \ldots \ldots \ldots \ldots \ldots+c_{n} x_{1}{ }^{n}\right) \sin \left(l x_{1}\right) \\
y_{2}=\left(c_{0}+c_{1} x_{2}+c_{2} x_{2}{ }^{2}+c_{3} x_{2}{ }^{3}\right. \\
\left.\ldots \ldots \ldots \ldots \ldots \ldots \ldots \ldots+c_{n} x_{2}{ }^{n}\right) \sin \left(l x_{2}\right) \\
y_{3}=\left(c_{0}+c_{1} x_{3}+c_{2} x_{3}{ }^{2}+c_{3} x_{3}{ }^{3}\right. \\
\left.\ldots \ldots \ldots \ldots \ldots \ldots \ldots \ldots+c_{n} x_{3}{ }^{n}\right) \sin \left(l x_{3}\right)
\end{gathered}
$$$$
\begin{aligned}
& y_{n}=\left(c_{0}+c_{1} x_{n}+c_{2} x_{n}{ }^{2}+c_{3} x_{n}{ }^{3}\right. \\
& \left.\ldots \ldots \ldots \ldots \ldots \ldots \ldots \ldots \ldots+c_{n} x_{n}{ }^{n}\right) \sin \left(l x_{n}\right)
\end{aligned}
$$

Which can be written in the matrix form as

$$
\begin{gathered}
{\left[\begin{array}{c}
y_{1} \\
y_{2} \\
y_{3} \\
\vdots \\
y_{n+1}
\end{array}\right]=} \\
{\left[\begin{array}{cccc}
\sin \left(l x_{1}\right) & x_{1} \sin \left(l x_{1}\right) & \ldots & x_{1}{ }^{n} \sin \left(l x_{1}\right) \\
\sin \left(l x_{2}\right) & x_{2} \sin \left(l x_{2}\right) & \ldots & x_{2}{ }^{n} \sin \left(l x_{2}\right) \\
\vdots & \vdots & \vdots & \vdots \\
\sin \left(l x_{n}\right) & x_{n} \sin \left(l x_{n}\right) & \ldots & x_{n}{ }^{n} \sin \left(l x_{n}\right)
\end{array}\right]\left[\begin{array}{c}
c_{0} \\
c_{1} \\
c_{3} \\
\vdots \\
c_{n}
\end{array}\right]} \\
\cong A X=B(\text { say })
\end{gathered}
$$

Where,

$$
\begin{gathered}
A=\left[\begin{array}{cccccccc}
1 & x_{1} & x_{1}{ }^{2} & x_{1}{ }^{3} & x_{1}{ }^{4} & \ldots \ldots \ldots \ldots & x_{1}{ }^{n} \\
1 & x_{2} & x_{2}{ }^{2} & x_{2}{ }^{3} & x_{2}{ }^{4} & \ldots \ldots \ldots \ldots & x_{2}{ }^{n} \\
1 & x_{3} & x_{3}{ }^{2} & x_{3}{ }^{3} & x_{3}{ }^{4} & \ldots \ldots \ldots \ldots & x_{3}{ }^{n} \\
& \multicolumn{1}{c}{\ldots \ldots \ldots \ldots} & \ldots & & \\
1 & x_{n} & x_{n}{ }^{2} & x_{n^{3}}{ }^{3} & x_{n}{ }^{4} & \ldots \ldots \ldots & x_{n}{ }^{n}
\end{array}\right] \\
X=\left[\begin{array}{c}
c_{0} \\
c_{1} \\
c_{3} \\
\vdots \\
c_{n}
\end{array}\right] \quad B=\left[\begin{array}{c}
y_{1} \\
y_{2} \\
y_{3} \\
\vdots \\
y_{n}
\end{array}\right]
\end{gathered}
$$

To obtained the solutions of arbitrary constants, we write,

$$
A^{T}(A X)=A^{T} B
$$

Where $A^{T}$ is the transpose matrix of A.

$$
\begin{gathered}
\text { Values of } A^{T} A=\left(a_{i j}\right)_{(n+1) \times(n+1)} \\
\text { and } A^{T} B=\left(b_{i}\right)_{(n+1) \times 1}
\end{gathered}
$$

$$
\text { such that } a_{i j}=\sum_{k=1}^{m}\left[x_{k}^{i+j-2}\left\{\sin \left(l x_{k}\right)\right\}^{2}\right]
$$

$$
\text { and } b_{i}=\sum_{k=1}^{m}\left[y_{k} x_{k}^{i} \sin \left(l x_{k}\right)\right], 1 \leq i \leq(n+1)
$$

$\mathrm{n}=$ order of the polynomial, $\mathrm{m}=$ no of collection of points, $x_{i}$ and $y_{i}$ ordinates and the absciss a of the given points.

Solution of the arbitrary constants $c_{0}, c_{1}, c_{2}, \ldots \ldots \ldots \ldots \ldots \ldots \ldots, c_{n}$ can be obtained with the help of Gauss-elimination method of general form

$$
\begin{aligned}
& R_{i}^{\prime} \rightarrow R_{i}-\frac{R_{j}}{a_{(i-1)(i-1)}} \times a_{i j} \\
& (2 \leq i \leq n+1),(1 \leq j \leq n)
\end{aligned}
$$

Steps of transformation will be done as follows:

$$
(2 \leq i \leq n+1 \text { when } j=1)
$$




$$
\begin{gathered}
(3 \leq i \leq n+1 \text { when } j=2) \\
\cdots \ldots \ldots \cdots \cdots \cdots \cdots \cdots \cdots \cdots \cdots \cdots \cdots \cdots \cdots \cdots \\
(n+1 \leq i \leq n+1 \text { when } j=n)
\end{gathered}
$$

These operations help us to get upper triangular matrix. By backward substitution we will get the solution of the mentioned arbitrary constants.

Thus any polynomial can be obtained into controllable form by multiplication of sine and cosine function.

For the different value of $l$ we will get different value of the constants. Because of different value of the constants we will have different imprecise functions. This characteristic of function is very important in the field of applications.

\section{A. Sine imprecise Polynomial of degree one-}

Example-1 (Sine imprecise function of polynomial of degree one with angle, $x)$ :

Let $y=c_{0}+c_{1} x$ be a polynomial of degree one. So, in particular

$$
y=\left(c_{0}+c_{1} x\right) \sin (x), \text { for } l=1
$$

is a sine imprecise polynomial of degree one.

Let us consider $(1,3.2), \quad(2,4),(3,-2.5), \quad(4,-$ $4.5),(5,6),(6,-7.5),(7,5.5)$ be the points in the data collection which are above and below the $\mathrm{x}$-axis .

$$
\begin{gathered}
\text { So, } x_{1}=1, x_{2}=2, x_{3}=3, x_{4}=4, \\
x_{5}=5, x_{6}=6, x_{7}=7 \\
y_{1}=3.2, y_{2}=4, y_{3}=-2.5 \\
y_{4}=-4.5, y_{5}=6, y_{6}=-7.5, y_{7}=5.5
\end{gathered}
$$

By the law of roots the given imprecise function satisfies the given collection of points to have linear simultaneous equation to form a matrix as follows:

From (4), (5), (6) (10), we have

$$
\begin{gathered}
A X=B \\
=>\left(A^{T} A\right) X=A^{T} B
\end{gathered}
$$

Where, $A^{T} A=\left(a_{i j}\right)_{(n+1) \times(n+1)}$

$$
A^{T} B=\left(a_{i j}\right)_{(n+1) \times 1}, \quad X=\left[\begin{array}{l}
c_{0} \\
c_{1}
\end{array}\right]
$$

Such that $a_{i j}=\sum_{k=1}^{m} x_{k}^{i+j-2}\left\{\sin \left(x_{k}\right)\right\}^{2}$

and $b_{i}=\sum_{k=1}^{m} y_{k} x_{k}^{i} \sin \left(x_{k}\right), 1 \leq i \leq(n+1)$

Here, $m=$ no. of points $=7, n=$ degree of the polynomial $=1$

$$
\begin{gathered}
A^{T} A=\left(a_{i j}\right)_{2 \times 2} \text { and } A^{T} B=\left(b_{i}\right)_{2 \times 1} \\
\text { Where } a_{i j}=\sum_{k=1}^{7} x_{k}^{i+j-2}\left\{\sin \left(x_{k}\right)\right\}^{2} \\
\text { and } b_{i}=\sum_{k=1}^{7} y_{k} x_{k}^{i} \sin \left(x_{k}\right), \quad 1 \leq i \leq 2
\end{gathered}
$$

$$
\begin{aligned}
& a_{11}=\sum_{k=1}^{7} x_{k}{ }^{0}\left\{\sin \left(x_{k}\right)\right\}^{2} \\
& =\sin ^{2}\left(x_{1}\right)+\sin ^{2}\left(x_{2}\right)+\sin ^{2}\left(x_{3}\right)+\sin ^{2}\left(x_{4}\right) \\
& +\sin ^{2}\left(x_{5}\right)+\sin ^{2}\left(x_{6}\right)+\sin ^{2}\left(x_{7}\right) \\
& =0.04250 \text {, } \\
& a_{12}=a_{21}=\sum_{k=1}^{7} x_{k}\left\{\sin \left(x_{k}\right)\right\}^{2} \\
& =x_{1} \sin ^{2}\left(x_{1}\right)+x_{2} \sin ^{2}\left(x_{2}\right)+x_{3} \sin ^{2}\left(x_{3}\right)+x_{4} \sin ^{2}\left(x_{4}\right) \\
& +x_{5} \sin ^{2}\left(x_{5}\right)+x_{6} \sin ^{2}\left(x_{6}\right) \\
& +x_{7} \sin ^{2}\left(x_{7}\right) \\
& =0.23883 \\
& a_{22}=\sum_{k=1}^{7} x_{k}{ }^{2}\left\{\sin \left(x_{k}\right)\right\}^{2} \\
& =x_{1}{ }^{2} \sin ^{2}\left(x_{1}\right)+x_{2}{ }^{2} \sin ^{2}\left(x_{2}\right)+x_{3}{ }^{2} \sin ^{2}\left(x_{3}\right) \\
& +x_{4}{ }^{2} \sin ^{2}\left(x_{4}\right)+x_{5}{ }^{2} \sin ^{2}\left(x_{5}\right) \\
& +x_{6}{ }^{2} \sin ^{2}\left(x_{6}\right)+x_{7}{ }^{2} \sin ^{2}\left(x_{7}\right) \\
& =1.141868 \text {, } \\
& b_{1}=\sum_{k=1}^{7} y_{k} x_{k} \sin \left(x_{k}\right) \\
& =y_{1} x_{1} \sin \left(x_{1}\right)+y_{2} x_{2} \sin \left(x_{2}\right)+y_{3} x_{3} \sin \left(x_{3}\right) \\
& +y_{4} x_{4} \sin \left(x_{4}\right)+y_{5} x_{5} \sin \left(x_{5}\right) \\
& +y_{6} x_{6} \sin \left(x_{6}\right)+y_{7} x_{7} \sin \left(x_{7}\right) \\
& =1.345599 \\
& b_{2}=\sum_{k=1}^{7} y_{k} x_{k}{ }^{2} \sin \left(x_{k}\right) \\
& =y_{1} x_{1}{ }^{2} \sin \left(x_{1}\right)+y_{2} x_{2}{ }^{2} \sin \left(x_{2}\right)+y_{3} x_{3}{ }^{2} \sin \left(x_{3}\right) \\
& +y_{4} x_{4}{ }^{2} \sin \left(x_{4}\right)+y_{5} x_{5}{ }^{2} \sin \left(x_{5}\right) \\
& +y_{6} x_{6}{ }^{2} \sin \left(x_{6}\right)+y_{7} x_{7}{ }^{2} \sin \left(x_{7}\right) \\
& =12.10867 \\
& \text { Thus }\left[\begin{array}{ll}
0.04 & 0.23 \\
0.23 & 1.41
\end{array}\right]\left[\begin{array}{l}
c_{0} \\
c_{1}
\end{array}\right]=\left[\begin{array}{c}
1.34 \\
12.10
\end{array}\right]
\end{aligned}
$$

From the formula (11), we have

$$
\begin{aligned}
& R_{i}^{\prime} \rightarrow R_{i}-\frac{R_{j}}{a_{(i-1)(i-1)}} \times a_{i j} ;(2 \leq i \leq 2),(1 \leq j \leq 1) \\
& {\left[\begin{array}{cc}
0.04 & 0.23 \\
0 & 0.087
\end{array}\right]\left[\begin{array}{l}
c_{0} \\
c_{1}
\end{array}\right]=\left[\begin{array}{l}
1.28 \\
4.39
\end{array}\right]} \\
& =>0.04 c_{0}+0.23 c_{1}=1.28,0.087 c_{1}=4.39 \\
& \text { So, } c_{1}=\frac{4.39}{0.087}=50.45 \text {, } \\
& c_{0}=\frac{1}{0.04}\left(1.28-0.23 c_{1}\right) \\
& =\frac{1}{0.04}(1.28-0.23 \times 50.45)=-258.08 \\
& \text { So, } y=(-258.08+50.45 x) \sin (x)
\end{aligned}
$$




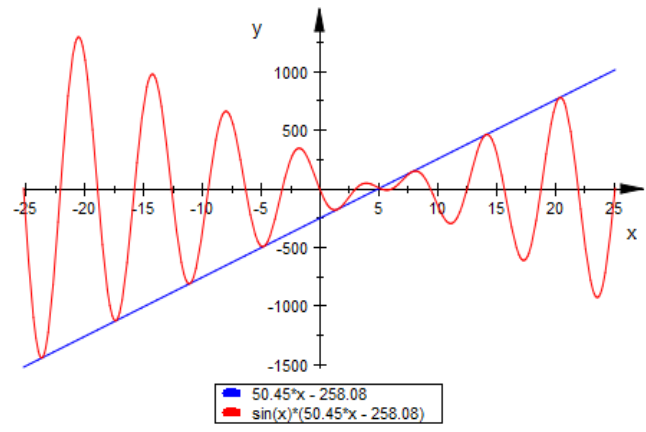

Fig.1. Graph of $y=-258.08+50.45 x$ and $y=(-258.08+$ $50.45 \mathrm{x}) \sin (\mathrm{x})$

Here, $\frac{258.08}{50.45}=5.115$ and $\pi<5.115<2 \pi$, for which $x=\pi$ and $2 \pi$ are the nearest two values such that $\sin (x)=0$

So, the graph starts to oscillate from the point, $x=\frac{5.115+2 \pi}{2} \simeq 5.699$ (approx.) along the positive $\mathrm{x}$ axis and $x=\frac{5.115+\pi}{2} \simeq 4.128$ (approx). along the negative $x$-axis which is shown in the Fig 1 .

So,

$$
\left(\frac{5.115+2 \pi}{2},\left(-258.08+50.45\left(\frac{5.115+2 \pi}{2}\right)\right) \sin \left(\frac{5.115+2 \pi}{2}\right)\right)
$$

is called conversion point along the positive $\mathrm{x}$-axis .

Example-2 (Sine imprecise function of polynomial of degree one with angle, $2 x)$ :

Let $y=c_{0}+c_{1} x$ be a polynomial of degree one. So, in particular

$$
y=\left(c_{0}+c_{1} x\right) \sin (2 x), \text { for } l=2
$$

is an imprecise polynomial of degree one.

Let us consider the same example taken for the equation (12)

Here, $\quad(1,3.2), \quad(2,4),(3,-2.5),(4,-4.5),(5,6),(6,-$ $7.5),(7,5.5)$ are the points in the data collection which are above and below the $\mathrm{x}$-axis.

$$
\begin{gathered}
\text { So, } x_{1}=1, x_{2}=2, x_{3}=3, x_{4}=4, \\
x_{5}=5, x_{6}=6, x_{7}=7 \\
y_{1}=3.2, y_{2}=4, y_{3}=-2.5 \\
y_{4}=-4.5, y_{5}=6, y_{6}=-7.5, y_{7}=5.5
\end{gathered}
$$

By the law of roots the given imprecise function satisfies the given collection of points to have linear simultaneous equation to form a matrix as follows:

From (4),(5),(6). (10),we have

$$
\begin{gathered}
A X=B \\
=>\left(A^{T} A\right) X=A^{T} B
\end{gathered}
$$

Where, $A^{T} A=\left(a_{i j}\right)_{(n+1) \times(n+1)}$

$$
A^{T} B=\left(b_{i}\right)_{(n+1) \times 1}, \quad X=\left[\begin{array}{l}
c_{0} \\
c_{1}
\end{array}\right]
$$

Such that $a_{i j}=\sum_{k=1}^{m} x_{k}^{i+j-2}\left\{\sin \left(2 x_{k}\right)\right\}^{2}$ and $b_{i}=$ $\sum_{k=1}^{m} y_{k} x_{k}^{i} \sin \left(2 x_{k}\right), 1 \leq i \leq(n+1)$

Here, $m=n o$. of points $=7$ and $n=$ degree of the polynomial $=1$

$$
A^{T} A=\left(a_{i j}\right)_{2 \times 2} \text { and } A^{T} B=\left(a_{i j}\right)_{2 \times 1}
$$

Where $a_{i j}=\sum_{k=1}^{7} x_{k}^{i+j-2}\left\{\sin \left(2 x_{k}\right)\right\}^{2}$ and $b_{i}=$ $\sum_{k=1}^{7} y_{k} x_{k}^{i} \sin \left(2 x_{k}\right), 1 \leq i \leq 2$

$$
\begin{aligned}
& a_{11}=\sum_{k=1}^{7} x_{k}{ }^{0}\left\{\sin \left(2 x_{k}\right)\right\}^{2} \\
& =\sin ^{2}\left(2 x_{1}\right)+\sin ^{2}\left(2 x_{2}\right)+\sin ^{2}\left(2 x_{3}\right)+\sin ^{2}\left(2 x_{4}\right) \\
& +\sin ^{2}\left(2 x_{5}\right)+\sin ^{2}\left(2 x_{6}\right)+\sin ^{2}\left(2 x_{7}\right) \\
& =0.16828 \text {, } \\
& a_{12}=a_{21}=\sum_{k=1}^{7} x_{k}\left\{\sin \left(2 x_{k}\right)\right\}^{2} \\
& =x_{1} \sin ^{2}\left(2 x_{1}\right)+x_{2} \sin ^{2}\left(2 x_{2}\right)+x_{3} \sin ^{2}\left(2 x_{3}\right) \\
& +x_{4} \sin ^{2}\left(2 x_{4}\right)+x_{5} \sin ^{2}\left(2 x_{5}\right) \\
& +x_{6} \sin ^{2}\left(2 x_{6}\right)+x_{7} \sin ^{2}\left(2 x_{7}\right) \\
& =0.94102 \text {, } \\
& a_{22}=\sum_{k=1}^{7} x_{k}{ }^{2}\left\{\sin \left(2 x_{k}\right)\right\}^{2} \\
& =x_{1}{ }^{2} \sin ^{2}\left(2 x_{1}\right)+x_{2}{ }^{2} \sin ^{2}\left(2 x_{2}\right)+x_{3}{ }^{2} \sin ^{2}\left(2 x_{3}\right) \\
& +x_{4}{ }^{2} \sin ^{2}\left(2 x_{4}\right)+x_{5}{ }^{2} \sin ^{2}\left(2 x_{5}\right) \\
& +x_{6}{ }^{2} \sin ^{2}\left(2 x_{6}\right)+x_{7}^{2} \sin ^{2}\left(2 x_{7}\right) \\
& =5.60673 \text {, } \\
& b_{1}=\sum_{k=1}^{7} y_{k} x_{k} \sin \left(x_{k}\right) \\
& =y_{1} x_{1} \sin \left(2 x_{1}\right)+y_{2} x_{2} \sin \left(2 x_{2}\right)+y_{3} x_{3} \sin \left(2 x_{3}\right) \\
& +y_{4} x_{4} \sin \left(2 x_{4}\right)+y_{5} x_{5} \sin \left(2 x_{5}\right) \\
& +y_{6} x_{6} \sin \left(2 x_{6}\right)+y_{7} x_{7} \sin \left(2 x_{7}\right) \\
& =2.49223 \\
& b_{2}=\sum_{k=1}^{7} y_{k} x_{k}^{2} \sin \left(x_{k}\right) \\
& =y_{1} x_{1}{ }^{2} \sin \left(2 x_{1}\right)+y_{2} x_{2}{ }^{2} \sin \left(2 x_{2}\right)+y_{3} x_{3}{ }^{2} \sin \left(2 x_{3}\right) \\
& +y_{4} x_{4}{ }^{2} \sin \left(2 x_{4}\right)+y_{5} x_{5}{ }^{2} \sin \left(2 x_{5}\right) \\
& +y_{6} x_{6}{ }^{2} \sin \left(2 x_{6}\right)+y_{7} x_{7}^{2} \sin \left(2 x_{7}\right) \\
& =23.96444 \\
& \text { Thus }\left[\begin{array}{ll}
0.16 & 0.94 \\
0.94 & 5.60
\end{array}\right]\left[\begin{array}{l}
c_{0} \\
c_{1}
\end{array}\right]=\left[\begin{array}{c}
2.49 \\
23.96
\end{array}\right]
\end{aligned}
$$

From the formula (11),we have-

$$
\begin{gathered}
R_{i}^{\prime} \rightarrow R_{i}-\frac{R_{j}}{a_{(i-1)(i-1)}} \times a_{i j} ; \\
(2 \leq i \leq 2),(1 \leq j \leq 1) \\
{\left[\begin{array}{cr}
0.16 & 0.94 \\
0 & 0.07
\end{array}\right]\left[\begin{array}{l}
c_{0} \\
c_{1}
\end{array}\right]=\left[\begin{array}{l}
2.49 \\
9.33
\end{array}\right]}
\end{gathered}
$$

I.J. Intelligent Systems and Applications, 2016, 10, 31-43 
So, as above we get

$$
\begin{gathered}
=>0.16 c_{0}+0.94 c_{1}=2.49, \quad 0.07 c_{1}=9.33 \\
c_{1}=\frac{9.33}{0.07}=133.28, \\
c_{0}=\frac{1}{0.16}\left(2.49-0.94 c_{1}\right) \\
=\frac{1}{0.16}(2.49-0.94 \times 133.28)=-746.64 \\
\text { So, } y=(-746.64+133.28 x) \sin (2 x)
\end{gathered}
$$

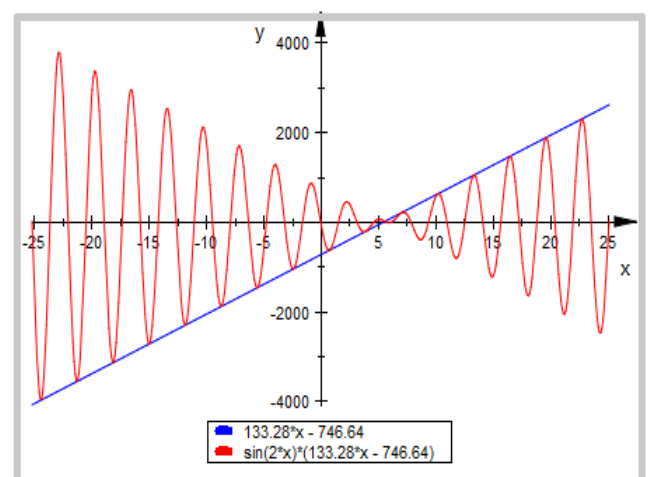

Fig.2. Graph of $=(-746.64+133.28 x)$ and $y=(-746.64+$ $133.28 \mathrm{x}) \sin (2 \mathrm{x})$

Here, $\frac{746.64}{133.28} \simeq 5.60$ and $\frac{3 \pi}{2}<5.60<2 \pi$ for which $x=\frac{3 \pi}{2}$ and $2 \pi$ are the nearest two values such that $\sin (2 x)^{2}=0$

So, the graph starts to oscillate from the point, $x=\frac{5.60+2 \pi}{2} \simeq 5.9423$ (approx.) along the positive $\mathrm{x}$ axis and $x=\frac{2 \times 5.60+3 \pi}{4} \simeq 5.156$ (approx.) along the negative $\mathrm{x}$-axis which is shown in the Fig 2 .

So,

$$
\left(\frac{5.60+2 \pi}{2},\left(\begin{array}{c}
-746.64+ \\
133.28\left(\frac{5.60+2 \pi}{2}\right)
\end{array}\right) \times \sin \left(2 \times \frac{5.60+2 \pi}{2}\right)\right)
$$

is called conversion point along the positive $\mathrm{x}$-axis .

Example-3 (Sine imprecise function of polynomial of degree one with angle, $3 x)$ :

Let $y=c_{0}+c_{1} x$ be a polynomial of degree one. So, in particular

$$
y=\left(c_{0}+c_{1} x\right) \sin (3 x), \text { for } l=3
$$

is an imprecise polynomial of degree one.

Let us consider the same example for the equation (12)

Here, $(1,3.2),(2,4),(3,-2.5),(4,-4.5),(5,6),(6,-7.5),(7,5.5)$ are the points in the data collection which are above and below the $\mathrm{x}$-axis.

$$
\begin{gathered}
\text { So, } x_{1}=1, x_{2}=2, x_{3}=3, x_{4}=4, \\
x_{5}=5, x_{6}=6, x_{7}=7 \\
y_{1}=3.2, y_{2}=4, y_{3}=-2.5,
\end{gathered}
$$

$$
y_{4}=-4.5, y_{5}=6, y_{6}=-7.5, y_{7}=5.5
$$

By the law of roots the given imprecise function satisfies the given collection of points to have linear simultaneous equation to form a matrix as follows:

From (4),(5),(6)...........(10),we have

$$
\begin{gathered}
A X=B \\
=>\left(A^{T} A\right) X=A^{T} B
\end{gathered}
$$

Where, $A^{T} A=\left(a_{i j}\right)_{(n+1) \times(n+1)}$

$$
A^{T} B=\left(b_{i}\right)_{(n+1) \times 1}, \quad X=\left[\begin{array}{l}
c_{0} \\
c_{1}
\end{array}\right]
$$

Such that $a_{i j}=\sum_{k=1}^{m} x_{k}^{i+j-2}\left\{\sin \left(3 x_{k}\right)\right\}^{2}$ and $b_{i}=$ $\sum_{k=1}^{m} y_{k} x_{k}^{i} \sin \left(3 x_{k}\right), 1 \leq i \leq(n+1)$

Here, $m=$ no. of points $=7$ and $n=$ degree of the polynomial $=1$

$$
A^{T} A=\left(a_{i j}\right)_{2 \times 2} \text { and } A^{T} B=\left(a_{i j}\right)_{2 \times 1}
$$

Where $a_{i j}=\sum_{k=1}^{7} x_{k}^{i+j-2}\left\{\sin \left(3 x_{k}\right)\right\}^{2}$ and $b_{i}=$ $\sum_{k=1}^{7} y_{k} x_{k}^{i} \sin \left(3 x_{k}\right), 1 \leq i \leq 2$

$$
\begin{gathered}
a_{11}=\sum_{k=1}^{7} x_{k}{ }^{0}\left\{\sin \left(3 x_{k}\right)\right\}^{2} \\
=\sin ^{2}\left(3 x_{1}\right)+\sin ^{2}\left(3 x_{2}\right)+\sin ^{2}\left(3 x_{3}\right)+\sin ^{2}\left(3 x_{4}\right) \\
+\sin ^{2}\left(3 x_{5}\right)+\sin ^{2}\left(3 x_{6}\right)+\sin ^{2}\left(3 x_{7}\right)
\end{gathered}
$$$$
=0.27677 \text {, }
$$$$
a_{12}=a_{21}=\sum_{k=1}^{7} x_{k}\left\{\sin \left(3 x_{k}\right)\right\}^{2}
$$$$
=x_{1}{ }^{2} \sin ^{2}\left(3 x_{1}\right)+x_{2}{ }^{2} \sin ^{2}\left(3 x_{2}\right)
$$$$
+x_{3}{ }^{2} \sin ^{2}\left(3 x_{3}\right)+x_{4}{ }^{2} \sin ^{2}\left(3 x_{4}\right)
$$$$
+x_{5}{ }^{2} \sin ^{2}\left(3 x_{5}\right)+x_{6}{ }^{2} \sin ^{2}\left(3 x_{6}\right)
$$$$
+x_{7}{ }^{2} \sin ^{2}\left(3 x_{7}\right)
$$$$
=2.077794
$$

$$
a_{22}=\sum_{k=1}^{7} x_{k}^{2}\left\{\sin \left(3 x_{k}\right)\right\}^{2}
$$$$
=x_{1}{ }^{2} \sin ^{2}\left(3 x_{1}\right)+x_{2}{ }^{2} \sin ^{2}\left(3 x_{2}\right)+x_{3}{ }^{2} \sin ^{2}\left(3 x_{3}\right)
$$$$
+x_{4}{ }^{2} \sin ^{2}\left(3 x_{4}\right)+x_{5}{ }^{2} \sin ^{2}\left(3 x_{5}\right)
$$$$
+x_{6}{ }^{2} \sin ^{2}\left(3 x_{6}\right)+x_{7}^{2} \sin ^{2}\left(3 x_{7}\right)
$$$$
=12.36365 \text {, }
$$

$$
\begin{gathered}
b_{1}=\sum_{k=1}^{7} y_{k} x_{k} \sin \left(3 x_{k}\right) \\
=y_{1} x_{1} \sin \left(3 x_{1}\right)+y_{2} x_{2} \sin \left(3 x_{2}\right)+y_{3} x_{3} \sin \left(3 x_{3}\right) \\
+y_{4} x_{4} \sin \left(3 x_{4}\right)+y_{5} x_{5} \sin \left(3 x_{5}\right) \\
+y_{6} x_{6} \sin \left(3 x_{6}\right)+y_{7} x_{7} \sin \left(3 x_{7}\right) \\
=3.744006
\end{gathered}
$$




$$
\begin{gathered}
b_{2}=\sum_{k=1}^{7} y_{k} x_{k}{ }^{2} \sin \left(3 x_{k}\right) \\
=y_{1} x_{1}{ }^{2} \sin \left(x_{1}\right)+y_{2} x_{2}{ }^{2} \sin \left(3 x_{2}\right)+y_{3} x_{3}{ }^{2} \sin \left(3 x_{3}\right) \\
+y_{4} x_{4}{ }^{2} \sin \left(3 x_{4}\right)+y_{5} x_{5}{ }^{2} \sin \left(3 x_{5}\right) \\
+y_{6} x_{6}{ }^{2} \sin \left(3 x_{6}\right)+y_{7} x_{7}{ }^{2} \sin \left(3 x_{7}\right) \\
=35.319439 \\
\text { Thus }\left[\begin{array}{cc}
0.27 & 2.07 \\
2.07 & 12.36
\end{array}\right]\left[\begin{array}{c}
c_{0} \\
c_{1}
\end{array}\right]=\left[\begin{array}{c}
3.74 \\
35.31
\end{array}\right]
\end{gathered}
$$

From the formula (11),we have-

$$
\begin{gathered}
R_{i}^{\prime} \rightarrow R_{i}-\frac{R_{j}}{a_{(i-1)(i-1)}} \times a_{i j} \\
(2 \leq i \leq 2),(1 \leq j \leq 1) \\
{\left[\begin{array}{cc}
0.27 & 2.07 \\
0 & -3.51
\end{array}\right]\left[\begin{array}{l}
c_{0} \\
c_{1}
\end{array}\right]=\left[\begin{array}{l}
3.74 \\
6.63
\end{array}\right]}
\end{gathered}
$$

So, as above we get

$$
\begin{gathered}
=>0.27 c_{0}+2.07 c_{1}=3.74,-3.51 c_{1}=6.63 \\
c_{1}=\frac{6.63}{-3.51}=-1.888, \\
c_{0}=\frac{1}{0.27}\left(3.74-2.07 c_{1}\right) \\
=\frac{1}{0.27}(3.74-2.07 \times(-1.88)) \\
=28.26
\end{gathered}
$$

So, $y=(28.26-1.88 x) \sin (3 x)$

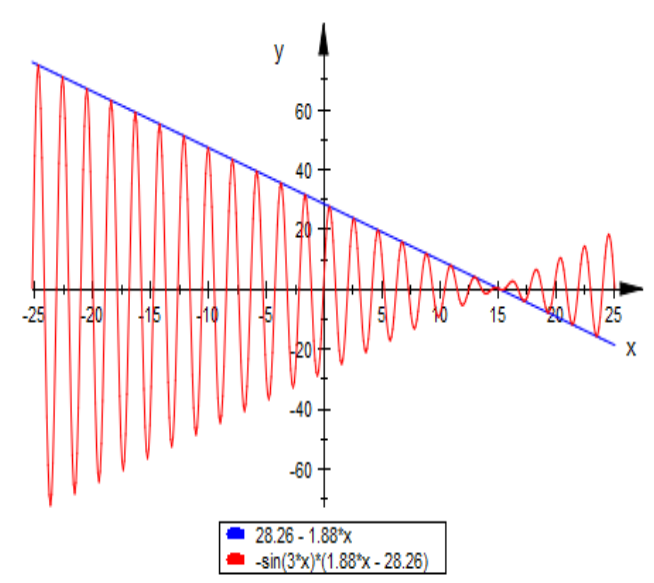

Fig. 3. Graph of $y=(28.26-1.88 x)$ and $y=(28.26-1.88 x) \sin (3 x)$

Here, $\frac{28.26}{1.88} \simeq 15.03$ (approx.) and $\frac{14 \pi}{3}<15.03<$ $5 \pi$, for which $x=\frac{14 \pi}{3}$ and $5 \pi$ are the nearest two values such that $\sin (3 x)=0$

So, the graph starts to oscillate from the point, $x=\frac{15.03+5 \pi}{2} \simeq 15.370$ (approx.) along the positive $\mathrm{x}$ axis, and $x=\frac{3 \times 15.03+14 \pi}{6} \simeq 14.837$ (approx.) along the negative $\mathrm{x}$-axis which is shown in the Fig 3 .

So,

$$
\left(\frac{15.03+5 \pi}{2},\left(28.26-1.88\left(\frac{15.03+5 \pi}{2}\right)\right) \sin \left(3 \times \frac{15.03+5 \pi}{2}\right)\right) \mathrm{m}
$$

is called conversion point along the positive $\mathrm{x}$-axis.

B. Sine imprecise function of polynomial of degree two:

Let $y=c_{0}+c_{1} x+c_{1} x^{2}$ be a polynomial of degree two. So, in particular

$$
y=\left(c_{0}+c_{1} x+c_{1} x^{2}\right) \sin (x), \text { for } l=1
$$

is an imprecise polynomial of degree two.

Let us consider $(1,0.5),(2,2.5),(3,2),(4,4)$,

$(5,3.5),(6,6),(7,7)$ be the points in the data collection.

$$
\begin{gathered}
\text { So, } x_{1}=1, x_{2}=2, x_{3}=3, \\
x_{4}=4, x_{5}=5, x_{6}=6, x_{7}=7 \\
y_{1}=0.5, y_{2}=2.5, y_{3}=2, \\
y_{4}=4, y_{5}=3.5, y_{6}=6, y_{7}=5.5
\end{gathered}
$$

From (4),(5),(6) ..(10),we have

$$
\begin{gathered}
A X=B \\
=>\left(A^{T} A\right) X=A^{T} B \\
\text { Where } A^{T} A=\left(a_{i j}\right)_{(n+1) \times(n+1)} \\
\text { and } A^{T} B=\left(b_{i 1}\right)_{(n+1) \times 1}
\end{gathered}
$$

Such that $a_{i j}=\sum_{k=1}^{m} x_{k}^{i+j-2}\left\{\sin \left(x_{k}\right)\right\}^{2} \quad$ and $b_{i 1}=$ $\sum_{k=1}^{m} y_{k} x_{k}^{i} \sin \left(x_{k}\right), 1 \leq i \leq(n+1)$

Here, $m=n o$. of points $=7$ and $n=$ degree of the polynomial $=2$

$$
\begin{gathered}
A^{T} A=\left(a_{i j}\right)_{3 \times 3} \text { and } A^{T} B=\left(a_{i j}\right)_{3 \times 1} \\
\text { Where } a_{i j}=\sum_{k=1}^{7} x_{k}^{i+j-2}\left\{\sin \left(x_{k}\right)\right\}^{2} \\
\text { and } b_{i}=\sum_{k=1}^{7} y_{k} x_{k}^{i} \sin \left(x_{k}\right), \quad 1 \leq i \leq 3 \\
a_{11}=\sum_{k=1}^{7} x_{k}^{0}\left\{\sin \left(x_{k}\right)\right\}^{2}=0.0148521, \\
a_{12}=\sum_{k=1}^{7} x_{k}\left\{\sin \left(x_{k}\right)\right\}^{2}=0.341889 \\
a_{13}=\sum_{k=1}^{7}\left[x_{k}^{2}\left\{\sin \left(x_{k}\right)\right\}^{2}\right]=1.418684 \\
a_{21}=\sum_{k=1}^{7} x_{k}\left\{\sin \left(x_{k}\right)\right\}^{2}=0.341889
\end{gathered}
$$

I.J. Intelligent Systems and Applications, 2016, 10, 31-43 


$$
\begin{aligned}
& a_{22}=\sum_{k=1}^{7} x_{k}{ }^{2}\left\{\sin \left(x_{k}\right)\right\}^{2}=1.1418684 \\
& a_{23}=\sum_{k=1}^{7} x_{k}{ }^{3}\left\{\sin \left(x_{k}\right)\right\}^{2}=8.799282 \\
& a_{31}=\sum_{k=1}^{7} x_{k}{ }^{2}\left\{\sin \left(x_{k}\right)\right\}^{2}=1.1418684 \\
& a_{32}=\sum_{k=1}^{7} x_{k}^{3}\left\{\sin \left(x_{k}\right)\right\}^{2}=8.799282 \\
& a_{33}=\sum_{k=1}^{7} x_{k}^{4}\left\{\sin \left(x_{k}\right)\right\}^{2}=56.055255
\end{aligned}
$$

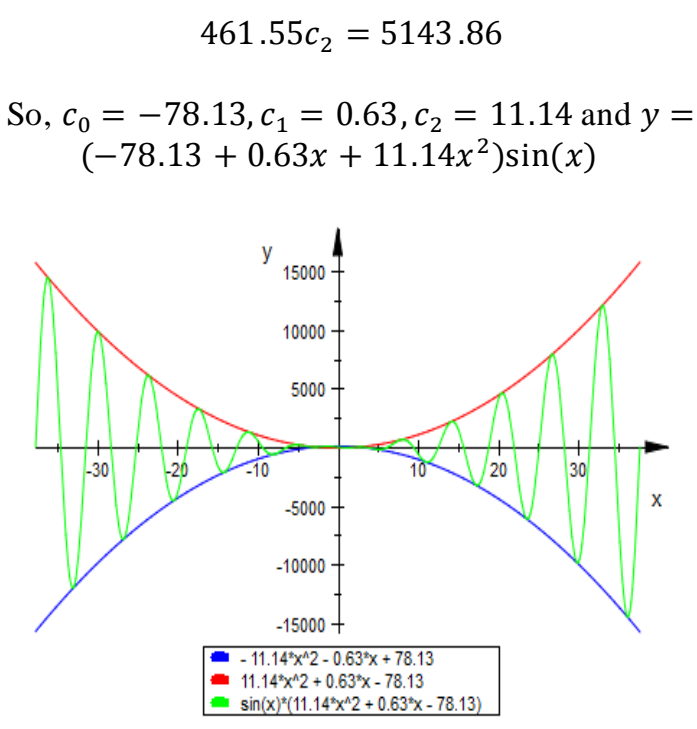

And

$$
\begin{aligned}
& b_{1}=\sum_{k=1}^{7} x_{k} y_{k} \sin \left(x_{k}\right)=11.593562 \\
& b_{2}=\sum_{k=1}^{7} x_{k}^{2} y_{k} \sin \left(x_{k}\right)=68.812246 \\
& b_{3}=\sum_{k=1}^{7} x_{k}^{3} y_{k} \sin \left(x_{k}\right)=424.896557
\end{aligned}
$$

Thus the simultaneous equation is converted into following simple matrix form:

$$
\left[\begin{array}{ccc}
0.015 & 0.34 & 1.14 \\
0.34 & 1.14 & 8.80 \\
1.14 & 8.80 & 56.06
\end{array}\right]\left[\begin{array}{l}
c_{0} \\
c_{1} \\
c_{1}
\end{array}\right]=\left[\begin{array}{c}
11.59 \\
68.81 \\
424.90
\end{array}\right]
$$

From the formula (11),we have-

$$
\begin{aligned}
& R_{i}^{\prime} \rightarrow R_{i}-\frac{R_{j}}{a_{(i-1)(i-1)}} \times a_{i j} \text {; } \\
& (2 \leq i \leq 3) ; j=1 \\
& \approx\left[\begin{array}{ccc}
0.015 & 0.34 & 1.14 \\
0 & -6.56 & -17.04 \\
0 & -17.04 & -30.58
\end{array}\right]\left[\begin{array}{l}
c_{0} \\
c_{1} \\
c_{1}
\end{array}\right] \\
& =\left[\begin{array}{c}
11.59 \\
-193.89 \\
-455.94
\end{array}\right] \\
& R_{i}^{\prime} \rightarrow R_{i}-\frac{R_{j}}{a_{(i-1)(i-1)}} \times a_{i j} ; \\
& (2 \leq i \leq 3) ; j=2 \\
& \approx\left[\begin{array}{ccc}
0.015 & 0.34 & 1.14 \\
0 & -6.59 & -17.04 \\
0 & 0 & 461.55
\end{array}\right]\left[\begin{array}{l}
c_{0} \\
c_{1} \\
c_{1}
\end{array}\right]=\left[\begin{array}{c}
11.59 \\
-193.8 \\
5143.86
\end{array}\right] \\
& =>0.015 c_{0}+0.34 c_{1}+1.14 c_{2}=11.59 \\
& =>-6.59 c_{1}-17.04 c_{2}=-193.98
\end{aligned}
$$

Fig.4. 2D graph of $y=\left(-78.13+0.63 x+11.14 x^{2}\right) \sin (x)$

Thus the solution is

$$
\begin{gathered}
x=\frac{0.63 \pm \sqrt{0.63^{2}+4 \times 78.13 \times 11.14}}{2 \times 78.13} \\
=0.38(\text { approx }),-0.37(\text { approx }) \\
\text { and }-\pi<-0.37<0 \text { and } 0<x<\pi
\end{gathered}
$$

So, the graph starts to oscillate from the point, $x=\frac{0.38+\pi}{2} \simeq 1.76$ (approx) along the positive $\mathrm{x}$-axis,

and $x=\frac{-0.37-\pi}{2} \simeq-1.75$ (approx) along the negative $\mathrm{x}$-axis which is shown in the Fig 4.

So,

$$
\begin{gathered}
\left(\frac{0.38+\pi}{2},\left(-78.13+0.63\left(\frac{0.38+\pi}{2}\right)+\right.\right. \\
\left.\left.11.14\left(\frac{0.38+\pi}{2}\right)^{2}\right) \sin \left(\frac{0.38+\pi}{2}\right)\right)
\end{gathered}
$$

is called conversion point along the positive $\mathrm{x}$-axis.

Further from the same point, imprecise function will come back to the orig inal function of polynomial when we remove the multip lication factors. So sometime it is called diversion point of the imprecise function with respect to the multiplication factor sine.

Thus from the above calculations, we have observed that if the angle of the trigonometry function is increased then conversion point come closer to the origin of the coordinate axis system. It means that considered experiment starts to oscillate within the short period of time to have short wave length and larger energy in the system.

For example if the wings of a Generator moves more angle then speed is increased and the energy output will be larger and larger due to short wave length and the closer conversion point.

\section{RATE EFFECT OF IMPRECISENESS FOR THE IMPRECISE FUNCTIONS}

It is the ratio of the volume occupied by the objects 
and the volume of the place where the object is used. This value gives us information for any places whether the design experiment is properly done or not. If it is not properly designed then we will modify it again and again. Normally one is the value of the rate effect of impreciseness for which an experiment well designed or not is counted. To study how per the behavior of the object is effecting to the medium, it is very much necessary to know area occupied by any particular object.

A. Area bounded by sine imprecise functions of polynomial of degree one:

To obtain area by the help of integration all the interval cannot be allowed to take as a limit for the imprecise function. For example-

$$
\begin{aligned}
& \int_{0}^{2 \pi}\left(c_{0}+c_{1} x\right) \sin (x) d x=0 \\
& \int_{0}^{\pi}\left(c_{0}+c_{1} x\right) \sin (2 x) d x=0
\end{aligned}
$$

etc. For this reason to obtain area of an imprecise function imprecise number will be taken as a limit of the integration. After defining area obtained by the imprecise number we will obtain area of the imprecise function for any interval in the summation form.

(i) Sine imprecise function of angle multiplication one:

Thus for an imprecise function,

$$
\text { for } l=1, y=\left(c_{0}+c_{1} x\right) \sin (x) \text {, }
$$

we have section of intervals, $\left[0, \frac{\pi}{2}, \pi\right],\left[\pi, \frac{3 \pi}{2}, 2 \pi\right]$,

$$
\left[2 \pi, \frac{5 \pi}{2}, 3 \pi\right],\left[3 \pi, \frac{7 \pi}{2}, 4 \pi\right]
$$

having indicator functions for as follows:

$$
\begin{aligned}
& \mu_{N}(x)=\left\{\begin{array}{l}
\emptyset(x) ; \quad 0 \leq x \leq \frac{n \pi}{2} \\
\varphi(x) ; \frac{n \pi}{2} \leq x \leq n \pi
\end{array}\right. \\
& \text { such that } \emptyset(0)=\varphi(n \pi)=0
\end{aligned}
$$

Here, the function has maximum level at the point $=\frac{n \pi}{2}$.

So, we can call, $\left[0, \frac{\pi}{2}, \pi\right],\left[\pi, \frac{3 \pi}{2}, 2 \pi\right],\left[2 \pi, \frac{5 \pi}{2}, 3 \pi\right]$, $\left[3 \pi, \frac{7 \pi}{2}, 4 \pi\right]$ are an imprecise number. Since the indicator function has membership function and the reference function for an imprecise number. The area of the imprecise function will be measured in our system separately for the respective function so that we can have better information how their character change for long interval in an imprecise function. Here the limit of the integrations are taken in between maximum and minimum point, as in between this two points the above mentioned membership function and the reference functions are counted. For this example let us define the area bounded by an imprecise function of degree one obtained by sine multiplication of function as follows:

$$
\begin{gathered}
I_{1}=\int_{0}^{\frac{\pi}{2}}\left(c_{0}+c_{1} x\right) \sin (x) d x=C_{0}+C_{1} \\
I_{2}=\int_{\frac{\pi}{2}}^{\pi}\left(c_{0}+c_{1} x\right) \sin (x) d x \\
\quad=C_{0}+C_{1}(\pi-1) \\
I_{3}=\int_{\pi}^{\frac{3 \pi}{2}}\left(c_{0}+c_{1} x\right) \sin (x) d x \\
=-C_{0}-C_{1}(\pi+1) \\
I_{4}=\int_{\frac{3 \pi}{2}}^{2 \pi}\left(c_{0}+c_{1} x\right) \sin (x) d x \\
=-C_{0}-C_{1}(2 \pi-1)
\end{gathered}
$$

$$
\begin{aligned}
I_{5}= & \int_{2 \pi}^{\frac{5 \pi}{2}}\left(c_{0}+c_{1} x\right) \sin (x) \mathrm{dx} \\
= & C_{0}+C_{1}(2 \pi+1)
\end{aligned}
$$

$$
\begin{aligned}
I_{6}= & \int_{\frac{5 \pi}{2}}^{3 \pi}\left(c_{0}+c_{1} x\right) \sin (x) d x \\
& =C_{0}+C_{1}(3 \pi-1)
\end{aligned}
$$

$$
\begin{aligned}
& I_{7}=\int_{3 \pi}^{\frac{7 \pi}{2}}\left(c_{0}+c_{1} x\right) \sin (x) d x \\
& =-C_{0}-C_{1}(3 \pi+1) \\
& I_{8}=\int_{\frac{7 \pi}{2}}^{4 \pi}\left(c_{0}+c_{1} x\right) \sin (x) d x \\
& =-C_{0}-C_{1}(4 \pi-1) \quad \text { so on. }
\end{aligned}
$$

Here, negative signs are shown area bounded by the imprecise function lower part of the $\mathrm{x}$-axis.

Area of the membership imprecise function $=I_{1}+$ $\left|I_{3}\right|+I_{5}+\left|I_{7}\right|=\frac{n}{2} c_{0}+c_{1} \sum_{k=1}^{\frac{n}{2}}[(k-1) \pi+1] ; \mathrm{n}=8$

I.J. Intelligent Systems and Applications, 2016, 10, 31-43 
Area of the reference imprecise function is

$$
I_{2}+\left|I_{4}\right|+I_{6}+\left|I_{8}\right|=\frac{n}{2} c_{0}+c_{1} \sum_{k=1}^{\frac{n}{2}}[k \pi-1], ; \mathrm{n}=8
$$

Area of this imprecise function for this interval is

$$
\begin{gathered}
I_{1}+I_{2}+\left|I_{3}\right|+\left|I_{4}\right|+I_{5}+\left|I_{7}\right|+\left|I_{8}\right| \\
=\int_{0}^{4 \pi}\left(c_{0}+c_{1} x\right) \sin (x) d x \\
=(2 \times 4 \times 1) c_{0}+c_{1} \sum_{k=1}^{4}[(2 k-1) \pi]
\end{gathered}
$$

Thus the area of this imprecise function in the interval $[0, \pi]$ is

$$
\begin{gathered}
I_{n}=\int_{0}^{n \pi}\left(c_{0}+c_{1} x\right) \sin (x) d x \\
=(2 n .1) c_{0}+c_{1} \sum_{k=1}^{n}[(2 k-1) \pi]
\end{gathered}
$$

This value is known as the membership value of the sine imprecise polynomial of degree one for the angle $x$.

Thus $\frac{I_{n}}{\text { Total area bouded by the experiment }} ; n \in N$ is called rate per imprecise number of the respective intervals.

So, $\frac{\sum I_{n}}{\text { Total area bouded by the experiment }} ; n \in N$ is called rate of convergence of the imprecise function.

If $\left\{\frac{\sum I_{n}}{\text { Total area bouded by the experiment }} ; n \in N\right\} \leq 1$, the system is completely controllable. Otherwise we have to modify this function to into the controllable form.

Variance of this function in the respective interval is $\sigma^{2}=\left[\frac{\left\{\sum I_{n}\right\}^{2}}{\text { no. of observation }}\right]$

Standard deviation,

$$
\sum \sqrt{\left(\frac{I_{n}}{\text { Tota area bouded by the experiment }} ; n \in N\right)^{2}}
$$

Here the variance is the variation of area in the function for the different intervals.

(ii) Sine imprecise function of angle multiplication two:

For an imprecise function,

$$
\text { for } l=2, y=\left(c_{0}+c_{1} x\right) \sin (2 x) \text {, }
$$

we have section of intervals,

$\left[0, \frac{\pi}{4}, \frac{\pi}{2}\right],\left[\frac{\pi}{2}, \frac{3 \pi}{4}, \pi\right],\left[2 \pi, \frac{5 \pi}{2}, 3 \pi\right],\left[3 \pi, \frac{7 \pi}{2}, 4 \pi\right]$

, having indicator functions for as follows:

$$
\mu_{N}(x)=\left\{\begin{array}{l}
\emptyset(x) ; 0 \leq x \leq \frac{n \pi}{4} \\
\varphi(x) ; \frac{n \pi}{4} \leq x \leq \frac{n \pi}{2}
\end{array}\right.
$$

$$
\begin{aligned}
\text { such that } \phi(0) & =\varphi\left(\frac{n \pi}{2}\right)=0 \\
\text { and } \varnothing\left(\frac{n \pi}{4}\right) & =\varphi\left(\frac{n \pi}{4}\right)
\end{aligned}
$$

Here, function has maximum level at the point $=\frac{n \pi}{2}$. So we can call,

$$
\left[0, \frac{\pi}{4}, \frac{\pi}{2}\right],\left[\frac{\pi}{2}, \frac{3 \pi}{4}, \pi\right],\left[2 \pi, \frac{5 \pi}{2}, 3 \pi\right],\left[3 \pi, \frac{7 \pi}{2}, 4 \pi\right] \ldots \ldots,
$$

are all an imprecise numbers. Now,

$$
\begin{aligned}
& I_{1}=\int_{0}^{\frac{\pi}{4}}\left(c_{0}+c_{1} x\right) \sin (2 x) d x=\frac{c_{0}}{2}+\frac{c_{1}}{4} \\
& I_{2}=\int_{\frac{\pi}{4}}^{\frac{\pi}{2}}\left(c_{0}+c_{1} x\right) \sin (2 x) d x \\
& =+\frac{c_{0}}{2}+\frac{c_{1}(\pi-1)}{4} \\
& I_{3}=\int_{\frac{\pi}{2}}^{\frac{3 \pi}{4}}\left(c_{0}+c_{1} x\right) \sin (x) d x \\
& =-\frac{c_{0}}{2}-\frac{c_{1}(\pi+1)}{4} \\
& I_{4}=\int_{\frac{3 \pi}{4}}^{\pi}\left(c_{0}+c_{1} x\right) \sin (2 x) d x \\
& =-\frac{c_{0}}{2}-\frac{c_{1}(2 \pi-1)}{4} \\
& I_{5}=\int_{\pi}^{\frac{5 \pi}{4}}\left(c_{0}+c_{1} x\right) \sin (2 x) d x \\
& =\frac{c_{0}}{2}+\frac{c_{1}(2 \pi+1)}{4} \\
& I_{6}=\int_{\frac{5 \pi}{4}}^{\frac{3 \pi}{2}}\left(c_{0}+c_{1} x\right) \sin (2 x) d x \\
& =\frac{c_{0}}{2}+\frac{c_{1}(3 \pi-1)}{4} \\
& \begin{aligned}
I_{7}= & \int_{\frac{3 \pi}{2}}^{\frac{7 \pi}{4}}\left(c_{0}+c_{1} x\right) \sin (2 x) d x \\
& =-\frac{c_{0}}{2}-\frac{c_{1}(3 \pi+1)}{4}
\end{aligned}
\end{aligned}
$$




$$
\begin{aligned}
I_{8} & =\int_{\frac{7 \pi}{4}}^{2 \pi}\left(c_{0}+c_{1} x\right) \sin (2 x) d x \\
& \left.=-\frac{c_{0}}{2}-\frac{c_{1}(4 \pi-1)}{4}\right) \text { so on. }
\end{aligned}
$$

Here, negative signs are shown area bounded by the imprecise function lower part of the x-axis.

Area of the membership imprecise function is $=I_{1}+$ $\left|I_{3}\right|+I_{5}+\left|I_{7}\right|$

$$
=\frac{n}{2} c_{0}+\frac{c_{1}}{2^{2}} \sum_{k=1}^{\frac{n}{2}}[(k-1) \pi+1] ; n=8
$$

Area of the reference imprecise function is

$$
\begin{gathered}
I_{2}+\left|I_{4}\right|+I_{6}+\left|I_{8}\right| \\
=\frac{n}{2} c_{0}+\frac{c_{1}}{2^{2}} \sum_{k=1}^{2}[k \pi-1] ; n=8
\end{gathered}
$$

Area of this imprecise function in this interval is

$$
\begin{gathered}
I_{1}+I_{2}+\left|I_{3}\right|+\left|I_{4}\right|+I_{5}+\left|I_{7}\right|+\left|I_{8}\right| \\
=\int_{0}^{2 \pi}\left(c_{0}+c_{1} x\right) \sin (2 x) d x \\
=\frac{(2 \times 2 \times 2) c_{0}}{2}+\frac{c_{1}}{2^{2}} \sum_{k=1}^{2 \times 2}[(2 k-1) \pi] \\
\int_{0}^{4 \pi}\left(c_{0}+c_{1} x\right) \sin (2 x) d x \\
=\frac{(2 \times 4 \times 2) c_{0}}{2}+\frac{c_{1}}{2^{2}} \sum_{k=1}^{2 \times 4}[(2 k-1) \pi]
\end{gathered}
$$

Thus the area of the imprecise the function in the interval $[0, \pi]$ is

$$
\begin{gathered}
I_{n}=\int_{0}^{n \pi}\left(c_{0}+c_{1} x\right) \sin (2 x) d x \\
=\frac{c_{0}}{2}(2 n \times 2)+\frac{c_{1}}{2^{2}} \sum_{k=1}^{2 n}[(2 k-1) \pi]
\end{gathered}
$$

This value is known as the membership value of the sine imprecise polynomial of degree one for the angle $2 x$.

Thus $\frac{I_{n}}{\text { Total area bouded by the experiment }} ; n \in N$ is called rate per imprecise number of the respective intervals.

So, $\frac{\sum I_{n}}{\text { Total area bouded by the experiment }} ; n \in N$ is called rate of convergence of the imprecise function.
If $\left\{\frac{\sum I_{n}}{\text { Total area bouded by the experiment }} ; n \in N\right\} \leq 1$, the system is completely controllable. Otherwise we have to modify this function to into the controllable form.

Variance of this function in the respective interval is $\sigma^{2}=\left[\frac{\left\{\sum I_{n}\right\}^{2}}{\text { no. of observation }}\right]$

Standard deviation,

$$
\sum \sqrt{\left(\frac{I_{n}}{\text { Tota area bouded by the experiment }} ; n \in N\right)^{2}}
$$

Here the variance is the variation of area in the function for the different intervals.

(iii) Sine imprecise function of angle multiplication three:

For an imprecise function,

$$
\text { for } l=3, y=\left(c_{0}+c_{1} x\right) \sin (3 x)
$$

we have section of intervals, $\left[0, \frac{\pi}{6}, \frac{\pi}{3}\right],\left[\frac{\pi}{3}, \frac{\pi}{2}, \frac{2 \pi}{3}\right]$, $\left[\frac{2 \pi}{3}, \frac{5 \pi}{6}, \pi\right],\left[\pi, \frac{7 \pi}{6}, \frac{4 \pi}{3}\right]$ , having indicator functions for as follows:

$$
\begin{aligned}
& \mu_{N}(x)=\left\{\begin{array}{l}
\emptyset(x) ; 0 \leq x \leq \frac{n \pi}{6} \\
\varphi(x) ; \frac{n \pi}{6} \leq x \leq \frac{n \pi}{3}
\end{array}\right. \\
& \text { such that } \varnothing(0)=\varphi\left(\frac{n \pi}{3}\right)=0
\end{aligned}
$$

Here, function has maximum level at the point $=\frac{n \pi}{2}$. So we can call, $\left[0, \frac{\pi}{6}, \frac{\pi}{3}\right],\left[\frac{\pi}{3}, \frac{\pi}{2}, \frac{2 \pi}{3}\right],\left[\frac{2 \pi}{3}, \frac{5 \pi}{6}, \pi\right]$, $\left[\pi, \frac{7 \pi}{6}, \frac{4 \pi}{3}\right]$ , are all an imprecise number. Now,

$$
\begin{gathered}
I_{1}=\int_{0}^{\frac{\pi}{6}}\left(c_{0}+c_{1} x\right) \sin (3 x) d x=\frac{c_{0}}{3}+\frac{c_{1}}{9} \\
I_{2}=\int_{\frac{\pi}{6}}^{\frac{\pi}{3}}\left(c_{0}+c_{1} x\right) \sin (3 x) d x \\
=\frac{c_{0}}{3}+\frac{c_{1}(\pi-1)}{9} \\
I_{3}=\int_{\frac{\pi}{3}}^{\frac{\pi}{2}}\left(c_{0}+c_{1} x\right) \sin (3 x) d x \\
=-\frac{c_{0}}{3}-\frac{c_{1}(\pi+1)}{9}
\end{gathered}
$$




$$
\begin{aligned}
& I_{4}=\int_{\frac{\pi}{2}}^{\frac{2 \pi}{3}}\left(c_{0}+c_{1} x\right) \sin (3 x) d x \\
& =-\frac{c_{0}}{3}-\frac{c_{1}(2 \pi-1)}{9} \\
& I_{5}=\int_{\frac{2 \pi}{3}}^{\frac{5 \pi}{6}}\left(c_{0}+c_{1} x\right) \sin (3 x) d x \\
& =\frac{c_{0}}{3}+\frac{c_{1}(2 \pi+1)}{9} \\
& I_{6}=\int_{\frac{5 \pi}{6}}^{\pi}\left(c_{0}+c_{1} x\right) \sin (3 x) d x \\
& =\frac{c_{0}}{3}+\frac{c_{1}(3 \pi-1)}{9} \\
& I_{7}=\int_{\pi}^{\frac{7 \pi}{6}}\left(c_{0}+c_{1} x\right) \sin (3 x) d x \\
& =-\frac{c_{0}}{3}-\frac{c_{1}(3 \pi+1)}{9} \\
& I_{8}=\int_{\frac{7 \pi}{6}}^{\frac{4 \pi}{3}}\left(c_{0}+c_{1} x\right) \sin (3 x) d x \\
& \left.=-\frac{c_{0}}{3}-\frac{c_{1}(4 \pi-1)}{9}\right) \text { so on. }
\end{aligned}
$$

Here, negative signs are shown area bounded by the imprecise function lower part of the $\mathrm{x}$-axis.

Area of the membership imprecise function is $=I_{1}+$ $\left|I_{3}\right|+I_{5}=\frac{n}{3} c_{0}+\frac{c_{1}}{3^{2}} \sum_{k=1}^{\frac{n}{2}}[(k-1) \pi+1] ; n=6$

Area of the reference imprecise function is

$$
I_{2}+\left|I_{4}\right|+I_{6}=\frac{n}{3} c_{0}+\frac{c_{1}}{3^{2}} \sum_{k=1}^{\frac{n}{2}}[k \pi-1] ; n=6
$$

Area of this imprecise function in the this interval is $I_{1}+I_{2}+\left|I_{3}\right|+\left|I_{4}\right|+I_{5}+I_{6}=$

$$
\begin{gathered}
\int_{0}^{\pi}\left(c_{0}+c_{1} x\right) \sin (3 x) d x \\
=\frac{(2 \times 1 \times 3) c_{0}}{3}+\frac{c_{1}}{3^{2}} \sum_{k=1}^{3 \times 1}[(2 k-1) \pi] \\
\int_{0}^{4 \pi}\left(c_{0}+c_{1} x\right) \sin (3 x) d x
\end{gathered}
$$

$$
=\frac{(2 \times 4 \times 3) c_{0}}{3}+\frac{c_{1}}{3^{2}} \sum_{k=1}^{3 \times 4}[(2 k-1) \pi]
$$

Thus the area of the imprecise function in the interval $[0, \pi]$ is

$$
\begin{gathered}
I_{n}=\int_{0}^{n \pi}\left(c_{0}+c_{1} x\right) \sin (3 x) d x \\
=\frac{c_{0}}{3}(2 n \times 3)+\frac{c_{1}}{3^{2}} \sum_{k=1}^{3 n}[(2 k-1) \pi] ; n \varepsilon R
\end{gathered}
$$

This value is known as the membership value of the sine imprecise polynomial of degree one for the angle $3 x$.

Thus $\frac{I_{n}}{\text { Total area bouded by the experiment }} ; n \in N$ is called rate per imprecise number of the respective intervals.

So, $\frac{\sum I_{n}}{\text { Total area bouded by the experiment }} ; n \in N$ is called rate of convergence of the imprecise function.

If $\left\{\frac{\sum I_{n}}{\text { Total area bouded by the experiment }} ; n \in N\right\} \leq 1$, the system is completely controllable. Otherwise we have to modify this function to into the controllable form.

Variance of this function in the respective interval is $\sigma^{2}=\left[\frac{\left\{\sum I_{n}\right\}^{2}}{\text { no. of observation }}\right]$

Standard deviation,

$$
\sum \sqrt{\left(\frac{I_{n}}{\text { Tota area bouded by the experiment }} ; n \in N\right)^{2}}
$$

Here the variance is the variation of area in the function for the different intervals.

Thus (21), (23) and (25) lead us to introduce a general formula for finding the area of sine imprecise function of degree one as follows:

$$
\begin{gathered}
\int_{0}^{n \pi}\left(c_{0}+c_{1} x\right) \sin (l x) d x \\
=\frac{c_{0}}{l}(2 n \times l)+\frac{c_{1}}{l^{2}} \sum_{k=1}^{l n}[(2 k-1) \pi] ; l \in Z
\end{gathered}
$$

Here for any imprecise number total no. of membership and the reference function is always 2 nl for $n \in R$ and $l \in Z$

\section{CONCLUSION}

Obtaining a general formula of imprecise function in various situations was the main objective of this article. So the general formula of sine imprecise function is defined with the help of finite numbers of data collection of points in the beginning. This formula is tested in the 
particular problems. Finally area of the sine imprecise function is obtained by the help of integration and summation. Here the limits of the integration is taken from the imprecise number of different interval within the imprecise function and proposed a new formula of area in the last part of the article.

\section{ACKNOWLEDGMENT}

The authors first would like to thank the CIT, Kokrajhar for providing available facilities to bring out this article. Secondly would like thank the Bodoland University for their encouragement to bring out this article. Finally would like to thank all the reviewers for their careful reading of this article and for their helpful comments which are helped to improve this work.

\section{REFERENCES}

[1] J. L. Krahula and J. F. Polhemus, Use of Fourier Series in the Finite Element Method, AIAA Journal, Vol. 6, No. 4 (1968), 726-728.

[2] E.O. Attinger, A. Anné and D.A. McDonald, Use of Fourier Series for the Analy sis of Biological Sy stems, The Biophysical Society. Published by Elsevier Inc., Vol. 6, No.3 (1966), 291-304.

[3] H. Akima, A New Method of Interpolation and Smooth Curve Fitting Based on Local Procedures, Journal of the ACM, Vol. 17, No. 4 (1970), 589-602.

[4] C. Zhu and F. W. Paul, A Fourier Series Neural Network and Its Application to System Identification, J. Dyn. Sys., Meas., Control Vol.117, No.3 (1995), 253-261.

[5] C. H. Cheng, A new Approach to Ranking Fuzzy Numbers by Distance Method, Fuzzy Sets and Systems, Vol. 95 (1998), 307-317.

[6] E.J. Ekpenyong, C.O. Omekara, Application of Fourier Series Analy sis To Temperature Data, Global Journal of Mathematical Sciences Vol. 7, No.1( 2008), 5-14.

[7] F. Toutounian and A. Ataei, A New Method for Computing Moore-Penrose Inverse Matrices, Journal of Computational and Applied Mathematics, Vol. 228, No. 1 (2009), 412-417.

[8] M. Kahm, grofitt: Fitting Biological Growth Curves with R, Journal of Statistical Software, Vol. 33, No.7 (2010).

[9] H.K. Baruah, Theory of Fuzzy Sets: Beliefs and Realities, I.J. Energy Information and Communications. Vol.2, No.2 (2011), 1-22.

[10] H.K. Baruah, Construction of Membership Function of a Fuzzy Number, ICIC Express Letters, Vol. 5, No.2 (2011), 545-549.

[11] M. Abbasian, H. S. Yazdi, ans A. V. Mazloom, Kernel Machine Based Fourier Series, I. J. of Advanced Science and Technology Vol. 33 (2011).

[12] H.K .Baruah, An introduction to the theory of imprecise Sets: The Mathematics of Partial Presence, J. Math. Computer Science, Vol. 2, No.2 (2012), 110-124.

[13] T. J. Neog and D. K. Sut, An Introduction to the Theory of Imprecise Soft Sets, I.J. Intelligent Systems and Applications, Vol.11 (2012), 75-83.

[14] S. Narayanamoorthy, S. Saranya, and S. Maheswari, A Method for Solving Fuzzy Transportation Problem (FTP) using Fuzzy Russell's Method, I.J. Intelligent Systems and Applications, Vol. 2 (2013), 71-75.

[15] S. Borgoyary, A Few Applications of Imprecise Numbers, I.J. Intelligent Systems and Applications, Vol. 7, No.8 (2015), 9-17.
[16] S. Borgoyary, An Introduction of Two and Three Dimensional Imprecise Numbers, I.J. Information Engineering and Electronic Business, Vol.7, No.5 (2015), 27-38.

\section{Authors' Profiles}

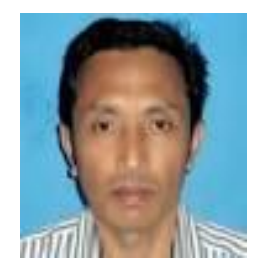

Dr. Kangujam Priyokumar Singh is a faculty of the Department of Mathematical Sciences, Bodoland University, Kokrajhar BTAD, Assam, India. He has published many articles in the field of Applied Mathematics in the reputed journals published under Springer, Elsevier and IOP.

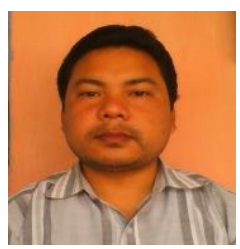

Sahalad Borgoyary is an Assistant Professor of the Central Institute of Technology Kokrajhar, BTAD, Assam, India and presently doing research in the Bodoland University under the guidance of Dr. K. Priyokumar Singh and Dr. H K Baruah. He has already published two articles in the international journal and another more articles are under review in the different journals. His research interests are included Fuzzy mathematics, Operations Research, Fractional Calculus etc.

How to cite this paper: Kangujam Priyokumar Singh, Sahalad Borgoyary, "Rate of Convergence of the Sine Imprecise Functions", International Journal of Intelligent Systems and Applications (IJISA), Vol.8, No.10, pp.31-43, 2016. DOI: 10.5815/ijisa.2016.10.04 